\title{
Development of a short food frequency questionnaire to assess diet quality in UK adults
}

\author{
S.R. Crozier ${ }^{1}$, S. Shaw ${ }^{1,2}$, H.M. Inskip ${ }^{1,2}$, J. Baird ${ }^{1,2}$, C. Cooper ${ }^{1,2}$ and C. Vogel ${ }^{1,2}$ \\ ${ }^{1}$ MRC Lifecourse Epidemiology Unit, University of Southampton, Southampton, UK and \\ ${ }^{2}$ NIHR Southampton Biomedical Research Centre, University of Southampton and University Hospital Southampton \\ NHS Foundation Trust, Southampton, UK
}

Food frequency questionnaires (FFQs) are a popular tool in nutritional epidemiology, enabling estimates of habitual diet in large populations, but are time-consuming to complete. There is an increasing need for a short, accurate dietary tool that characterises healthy dietary patterns for use in observational and interventional research.

The National Diet and Nutrition Survey (NDNS) randomly selected participants from across the UK and asked them to keep a diary of everything they ate and drank over four days. Diaries were coded and intake frequencies of foods derived using groups of nutritionally-similar foods. Principal component analysis (PCA) was performed on frequencies of consumption. Reduced-item prudent diet scores were calculated by multiplying coefficients for the fourteen most characteristic foods by each individual's standardized reported frequency of consumption. Both the full and reduced-item prudent diet scores were standardised. Willing participants provided a blood sample; beta carotene status was measured by high performance liquid chromatography and serum folate by liquid chromatography-mass spectrometry.

6090 adults (aged 20 to 96 years) provided dietary data in the first eight years of the NDNS (2008-2016). PCA of intake frequencies of 131 food groups revealed a prudent diet pattern characterised by high intakes of wholemeal bread, vegetables, fruit, water, oily fish and yoghurt, and low intakes of white bread, sugar, sugar-sweetened soft drinks and chips. The 14-item prudent diet score was calculated based on foods with the seven highest and seven lowest coefficients. Spearman's correlation between the full and 14-item prudent diet scores was 0.86 . The mean difference between the full and 14-item prudent diet scores was 0.00SDs with Bland-Altman limits of agreement -1.02 to $1.02 \mathrm{SDs}$. Notable correlations were seen between the full prudent diet score and beta-carotene $(\mathrm{rs}=0.45)$ and serum folate status $(r s=0.37)$; these were only slightly attenuated for the 14-item prudent diet score ( $r s=0.38, r s=0.33$ respectively).

A 14-item prudent diet score, based on foods that characterise the prudent dietary pattern, describes both a healthy and unhealthy diet. A 14-item FFQ would have clear advantages for time and resources, and may provide an appropriate tool to describe UK adult diets while reducing participant burden. 